\title{
Mean Value Estimation of Shape Operator on Triangular Meshes
}

\author{
Ahmed Fouad El Ouafdi ${ }^{1}$ \\ Research laboratory Labsiv, Faculty of Sciences \\ Ibn Zohr university, Agadir, Morocco
}

\author{
Hassan El Houari ${ }^{2}$ \\ LaGuardia Community College, \\ CUNY, New York, USA
}

\begin{abstract}
The principal curvatures, eigenvalues of the shape operator, are an important differential geometric features that characterize the object's shape, as a matter of fact, it plays a central role in geometry processing and physical simulation. The shape operator is a local operator resulting from the matrix quotient of normal derivative with the metric tensor, and hence, its matrix representation is not symmetric in general. In this paper, the local differential property of the shape operator is exploited to propose a local mean value estimation of the shape operator on triangular meshes. In contrast to the stat-of-art approximation methods that produce a symmetric operator, the resulting estimation matrix is accurate and generally not symmetric. Various comparative examples are presented to demonstrate the accuracy of proposed estimation. The results show that the principle curvature arising from the estimated shape operator are accurate in comparison with the standard estimation in the literature.
\end{abstract}

Keywords-Curvature estimation; shape operator; triangular meshes; discrete differential operator

\section{INTRODUCTION}

Surface curvatures are a significant intrinsic geometry component that describe the geometrical structure of a regular object surface. As the three-dimensional shape of objects is progressing substantially, measuring such curvatures is becoming more common in a variety of areas such as physics-based modeling, variational modeling, geometric data processing and computer graphics.

Although differential geometry has a longstanding heritage of computing curvatures on smooth surfaces, such curvatures, as well as other features, lose their continuity when a smooth surface is approximated by a triangle mesh due to the mesh's discrete nature. There is therefore the necessity to create approaches for the estimate of surface curvatures of triangular meshes, as precision and effectiveness remain the essential ingredients for the development of discrete evaluation methods. Over the last years, curvatures estimations problem has been extensively studied, since it is a crucial phase in mesh data processing due to its several applications in computer and robot vision, computer graphics, geometric modeling, and industrial and biomedical engineering [4], [13], [16], [17], [3]. Although there are various proposed methods for estimating curvature in the literature, the algorithm that aims for maximum estimation precision still always needed to be developed.

The shape operator, whose eigenvalues are the principal curvatures, has captivate a lot of attention since it is an essential ingredient to construct an accurate curvature estimate. In the smooth setting, computing a surface's shape operator is crucial, since the shape operator is equal to the gradient of the surface normal field. The first discretization of shape operator dates back to Taubin [18], who described the operator as a weighted average of normal curvatures. Since then, several other approaches to discretize shape operator on triangular meshes has been developed in the literature [2], [20], [7], [10], [22], [6], and most of these methods treated the shape operator as a local operator extracted from the matrices quotient of normal derivative with the metric tensor, which generally result a symmetrical matrix representation, even for object with unsymmetrical shape operator. In this paper, we use the local differential property of the shape operator to propose a convolutional based approach to estimate the shape operator on triangular meshes. The resulting estimation matrix is accurate and not necessarily symmetrical, unlike the stat-of-the-art approximation methods producing a symmetric operator.

\section{RElative WORK}

Curvatures estimation has been the subject of considerable research due to its several practical applications, leading to the development of a variety of curvature estimators. Most of the existing estimation approaches can be classified into two categories depending on whether the approach based on directional normal derivative approximation or local surface interpolation. In what follows, we briefly review some curvature estimation approaches from each category.

The first category estimate to shape operator on triangular face to develop an estimation of the curvature directly or through the curvature tensor. In [2], a discretization of the curvature surface tensor is based on the theory of normal cycles that estimates the curvature at the sampled smooth surfaces. Another approach based on degrees of freedom associated with normal vector is represented in [8], the curvature is estimated by formulating the shape operator from variational problems on general meshes. In [22], the finite difference approach is applied to discretize the directional derivative normal surface on each face. This method was later adopted by [1] using a collection of nearby sampling points combining the quadratic difference forms and the finite-difference normal directional derivative approximation. Another approach to estimating the surface's principal curvatures based on inversion-invariant local surface-based differential forms is proposed [23].

In [11], a per-face discrete curvature estimation approach is proposed in terms of discrete shape operator, the method is based on adapting the optimal estimation technique into a nonlinear diffusion process for normal and curvature consistencies. Another face-based method for estimating the curvature of 
triangle meshes focused on the concept of osculating circles in regular planes is discussed in [24]. More recently, a component analysis-based method is presented to estimate the curvatures in [25]. The approach identifies principal components that are dominant in the shape fields, resulting the first and second fundamental forms used in the curvature estimation.

The second category deals with determining the most accurate approximation of the surface patch for each data point neighborhood. In this direction, Theisel in [19] proposed a face-based approach for computing shape operators using linear interpolation of normal. In [21], an approach is introduced for estimating mean and Gaussian curvature and the shape operator matrix as well, it relies on the periodic structure of the normal curvatures to ensure that the quadrature are exact. In [14] a new method is proposed to estimate the curvature at different scales by adapting suitable fitting technique and applying it to different-sized neighborhood depending on scale. In [12] the interpolation of three end points and the corresponding normal vectors of each triangular vertex to construct a curved patch was introduced as a curvature estimate approach for meshes. In [15] a screen space method is proposed for estimating the mean and Gaussian curvature at interactive rates from the second fundamental form matrix by using positions and normal.

\section{A. Contribution}

The most proposed approximations of the shape operator are formulated in term of a symmetrical matrix that produces an inaccurate curvatures estimation, especially for object with unsymmetrical shape operator. As the shape operator is locally defined by a directional differential normal vector, we propose to estimate the shape operator by a mean value expression of normal difference at each vertex of triangular mesh. In contrast to the standard shape operator approximation methods, the resulting estimation matrix is generally not symmetric. We compare the principal curvatures, eigenvalues of the estimated shape operator, to the one arising from analytic expression. Various comparative examples are presented to demonstrate the accuracy of proposed curvatures estimation method.

The rest of the paper is organized as follows: In Section III we present a brief theoretical background that describes the construction of shape operator. In Section IV, we formulate the expression of the shape operator by neighborhood mean value formulation, and then we propose the new shape operator discretization algorithm whose evaluation through numerous substantiating examples is provided in Section V. We finally give some concluding remarks in Section VI.

\section{PRELIMINARY BACKGROUND}

In this section, we briefly review some definition related to the shape operator background, for more rigorous details, we refer interested reader to the standard differential geometry textbooks [5], [26].

Let us consider a smooth regular surface $\mathbb{M} \subseteq \mathbb{R}^{3}$ locally parameterized by $(x, y)$, where $T_{p} \mathbb{M}$ denoted the tangent plane of $\mathbb{M}$ at $p=p(x, y) \in \mathbb{M}$. The space $T_{p} \mathbb{M}$ is spanned by the partial derivatives $\mathrm{U}=\frac{\delta p}{\delta x}$ and $\mathrm{V}=\frac{\delta p}{\delta y}$ and it is equipped with

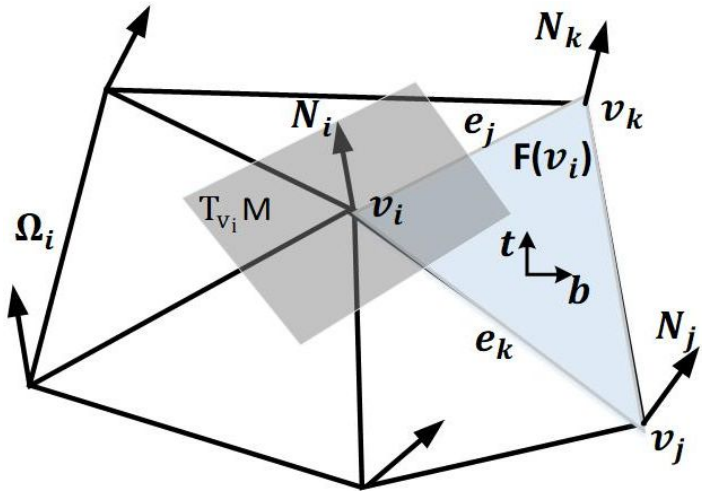

Fig. 1. Discrete Vertex Neighbourhood.

the standard inner product $(\cdot)$ in $\mathbb{R}^{3}$. The Riemannian metric tensor, called also first fundamental form, is defined as

$$
I=\left(\begin{array}{ll}
E & F \\
F & G
\end{array}\right)=\left(\begin{array}{ll}
\mathrm{U} \cdot \mathrm{U} & \mathrm{U} \cdot \mathrm{V} \\
\mathrm{V} \cdot \mathrm{U} & \mathrm{V} \cdot \mathrm{V}
\end{array}\right)
$$

The matrix $I$ is symmetric and positive definite. At every point $p(x, y) \in \mathbb{M}$, the unit normal vector field is defined by

$$
N(x, y)=\frac{\mathrm{U} \times \mathrm{V}}{|\mathrm{U} \times \mathrm{V}|}
$$

which allows to define the second fundamental form $I I=$ $I I(u, v)$ by

$$
I I=\left(\begin{array}{cc}
L & M \\
M & N
\end{array}\right)=\left(\begin{array}{cc}
\frac{\delta p}{\delta x \delta x} \cdot \mathrm{N} & \frac{\delta p}{\delta x \delta y} \cdot \mathrm{N} \\
\frac{\delta p}{\delta y \delta x} \cdot \mathrm{N} & \frac{\delta p}{\delta y \delta y} \cdot \mathrm{N}
\end{array}\right)
$$

or in term of normal directional derivative

$$
I I=-\left(\begin{array}{cc}
\frac{\delta p}{\delta x} \cdot \frac{\delta N}{\delta x} & \frac{\delta p}{\delta x} \cdot \frac{\delta N}{\delta y} \\
\frac{\delta p}{\delta y} \cdot \frac{\delta N}{\delta x} & \frac{\delta p}{\delta y} \cdots \frac{\delta N}{\delta y}
\end{array}\right)
$$

More generally, given a tangential vector $\mathrm{E} \in T_{p} \mathbb{M}$ at a point $p \in \mathbb{M}$, then the directional derivative $D_{\mathrm{E}} N$ of the the normal vector in the direction of vector $\mathrm{E}$ is defined by

$$
\mathcal{S}_{p}(\mathrm{E})=-D_{\mathrm{E}} N(p)
$$

where $\mathcal{S}: T_{p} \mathbb{M} \leftrightarrow T_{p} \mathbb{M}$ is a linear map called Shape operator that can be expressed in term of the first and second fundamental forms as

$$
S=I^{-1} I I=\left(\begin{array}{ll}
\frac{F M-G L}{E G-F^{2}} & \frac{F L-E M}{E G-F^{2}} \\
\frac{F N-G M}{E G-F^{2}} & \frac{F M-E N}{E G-F^{2}}
\end{array}\right)
$$

It should be noted that the above shape operator matrix need not to be symmetric in general, its eigenvalues, denoted with $k_{1}$ and $k_{2}$, are called the principal curvatures. The product and the mean of the principal curvatures are respectively called the Gaussian curvature $K=k_{1} k_{2}$ and the mean curvatures

$$
H=\frac{k_{1}+k_{2}}{2}
$$

In what follow, we propose a mean value based approach toward shape operator matrix estimation on triangular surfaces. 


\section{Shape Operator Estimation on Meshes}

For an arbitrary surface, the exact expression of the shape operator can rarely be expressed explicitly, hence, only an estimation on discrete surfaces can be performed. In the discrete setting, the surface $\mathbb{M}$ is sampled at $n_{p}$ points $P=$ $\left\{v_{1}, \ldots, v_{n_{p}}\right\}$. The points are then connected by $n_{e}$ edges $E=\left\{e_{1}, \ldots, e_{n_{e}}\right\}$ and $n_{f}$ faces $F=\left\{F_{1}, \ldots, F_{n_{f}}\right\}$ forming a triangular mesh $(V, E, F)$ noted $\mathcal{M}$. An orthogonal and normalized tangential reference frame $(\mathbf{t}, \mathbf{b})$ is attached to each triangle $f \in F$ as well as a normal vector $N_{f}$. As the shape operator is defined on a local surface, we consider a local discrete surface $\Omega_{i}=\cup F\left(v_{i}\right)$ around the point $v_{i} \in P$ as shown in Fig. 1. To estimate locally the shape operator at $v_{i}$ on the $\Omega_{i}$, we propose to use the following mean local value expression

$$
S_{v_{i}}=\frac{1}{\left|\Omega_{i}\right|} \int_{\Omega_{i}} \mathcal{S}_{v} d v
$$

where $\left|\Omega_{i}\right|$ denoted the area of the local surface $\Omega_{i}$. As $\Omega_{i}=$ $\cup F\left(v_{i}\right)$, where $F\left(v_{i}\right)$ is a face sharing the vertex $v_{i}$ depicted in Fig. 1, the local formulation of the shape operator (8) boils down to

$$
S_{v_{i}}=\frac{1}{\left|\Omega_{i}\right|} \sum_{F \in \Omega_{i}} \int_{F} S_{v} d v
$$

Hence, to estimate the shape operator over $\Omega_{i}$, it is sufficient to evaluate its expression on each incident face $F$. To this end, assume that the face $F\left(v_{i}, v_{j}, v_{k}\right)$ is determined by the three vertex $v_{i}, v_{j}$ and $v_{k}$. As the two vectors $e_{j}=v_{i}-v_{k}$ and $e_{k}=v_{j}-v_{i}$ are edges of the face $F$, hence, the two edges vectors can be fully expressed in the orthogonal frame $(\mathbf{t}, \mathbf{b})$ of $F$ as

$e_{j}=\underbrace{\left(e_{j} \cdot \mathbf{t}\right)}_{e_{j \mathbf{t}}} \mathbf{t}+\underbrace{\left(e_{j} \cdot \mathbf{b}\right)}_{e_{j \mathbf{b}}} \mathbf{b}$ and $e_{k}=\underbrace{\left(e_{k} \cdot \mathbf{t}\right)}_{e_{k \mathbf{t}}} \mathbf{t}+\underbrace{\left(e_{k} \cdot \mathbf{b}\right)}_{e_{k} \mathbf{b}} \mathbf{b}$

Using the expression of the shape operator in term of the normal directional derivative (5) along the two edges vector $e_{j}$ and $e_{k}$ give arise

$$
S_{v} \cdot e_{j}=-D_{e_{j}} N(v) \quad \text { and } \quad S_{v} \cdot e_{k}=-D_{e_{k}} N(v)
$$

Following Rusinkiewicz [22], we approximate the derivative of the normal vector $N$ in the direction of the two vector $e_{j}=v_{i}-v_{j}$ and $e_{k}=v_{k}-v_{i}$ on the face $F$ as

$$
\begin{gathered}
S_{v} \cdot e_{j}=-D_{e_{j}} N \approx\left(\begin{array}{c}
\left(N_{i}-N_{k}\right) \cdot \mathbf{t} \\
\left(N_{i}-N_{k}\right) \cdot \mathbf{b}
\end{array}\right) \text { and } \\
S_{v} \cdot e_{k}=-D_{e_{j}} N \approx\left(\begin{array}{c}
\left(N_{j}-N_{i}\right) \cdot \mathbf{t} \\
\left(N_{j}-N_{i}\right) \cdot \mathbf{b}
\end{array}\right)
\end{gathered}
$$

which leads to an approximation of the shape operator matrix $S_{v}$ of the normal vector $N$ inside the face $F$ as

$S(F) \approx\left(\begin{array}{ll}e_{j \mathbf{t}} & e_{j \mathbf{b}} \\ e_{k \mathbf{t}} & e_{j \mathbf{b}}\end{array}\right)^{-1}\left(\begin{array}{cc}\left(N_{i}-N_{k}\right) \cdot \mathbf{t} & \left(N_{i}-N_{k}\right) \cdot \mathbf{b} \\ \left(N_{j}-N_{i}\right) \cdot \mathbf{t} & \left(N_{j}-N_{i}\right) \cdot \mathbf{b}\end{array}\right)$

As the above estimation of the shape operator is locally constant over the face $F$, the integral formulation (9) can be expressed as

$$
S_{v_{i}}=\frac{1}{\left|\Omega_{i}\right|} \sum_{F \in \Omega_{i}}|F| \cdot S(F)
$$

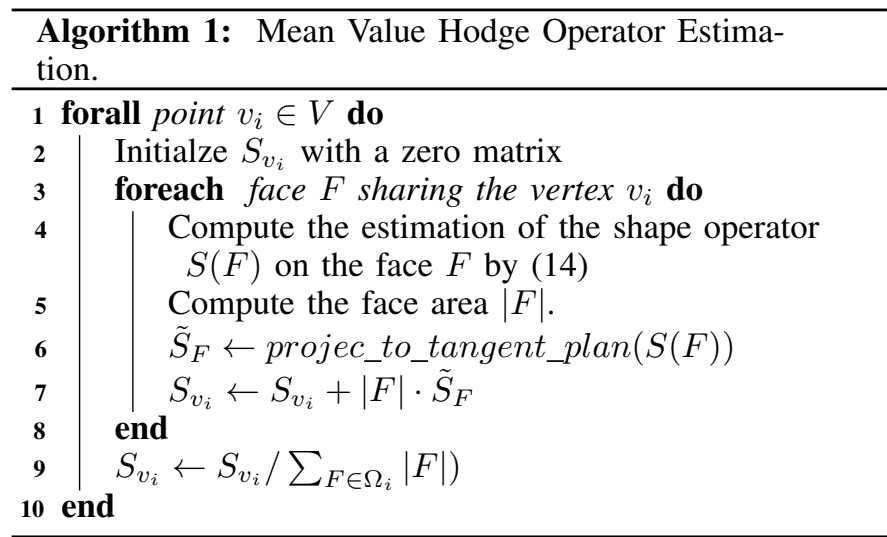

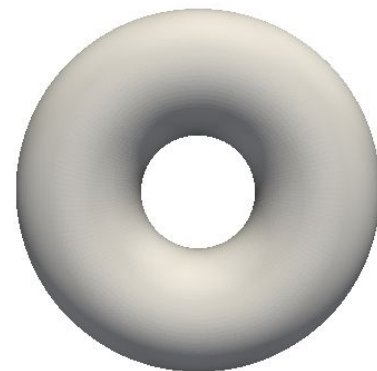

(a) Torus mesh

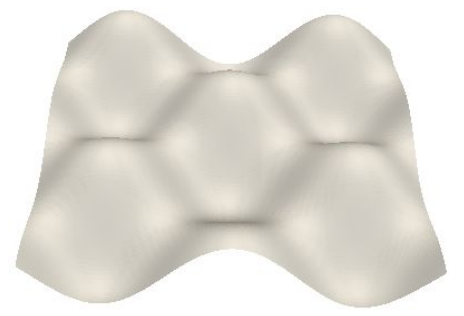

(b) Sinusoidal mesh
Fig. 2. Explicit Meshes Arising from Analytic Parametric Torus and Sinusoidal Surfaces.

The following algorithm summarize the shape operator estimation procedure on a triangular mesh: A per-processing step (10)of the algorithm 1 is to estimate the normal vector of each point $v_{i}$, then, we compute in the step 4 the shape operator (14) for each face $F$ incident to the vertex $v_{i}$, the obtained face based shape operator is projected back onto tangent plan of the vertex $v_{i}$ in the step 6 , and then summed up and normalized in the step 9 .

\section{EXPERIMENTAL RESULTS}

In the following, we report evaluation and comparison of the proposed Convolution Based Estimation algorithm (CBE) with three stat-of-art methods: the finite Difference curvature Estimation Method (DEM) proposed by Rusinkiewicz [22], the Multi-Scale Curvature (MSC) Estimation methods (CCM) [14] and the Normal Cycle Curvature (NCC) estimation method [2]. For each comparative method, the shape operator is estimated on a set of standard triangular meshes. In the following, a set of quantitative and qualitative comparison experiments of the four methods are performed.

\section{(14) Quantitative Evaluation}

In the first set of quantitative experiments, two explicit synthetic triangular meshes in Fig. 4 are selected, such that an analytic expression of the shape operator is available. The first mesh reported in Fig. 2(a) is the torus object with major radius 2 and minor radius 1 , the analytic expression of the shape operator of torus is represented by a symmetric matrix [26]. 


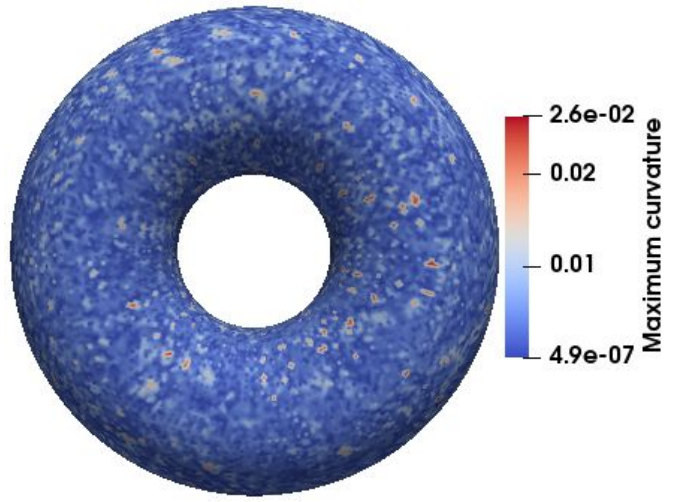

(a) CBE result

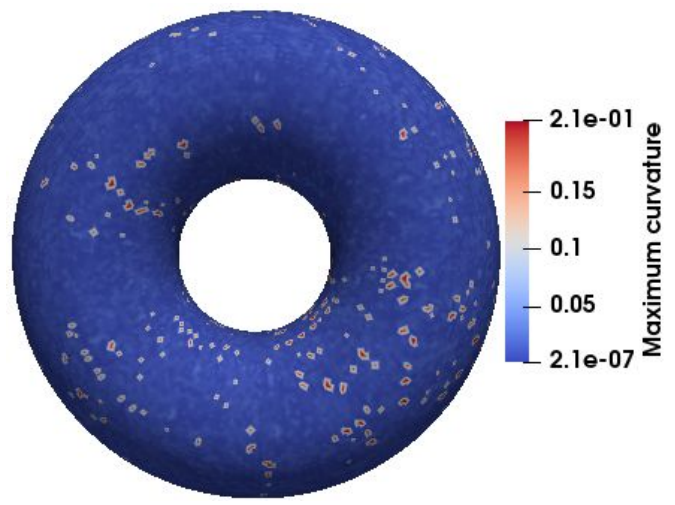

(b) DEM result

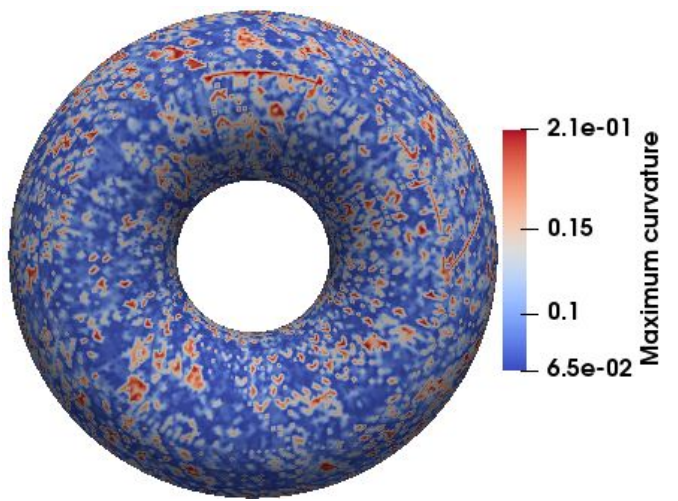

(c) MSC result

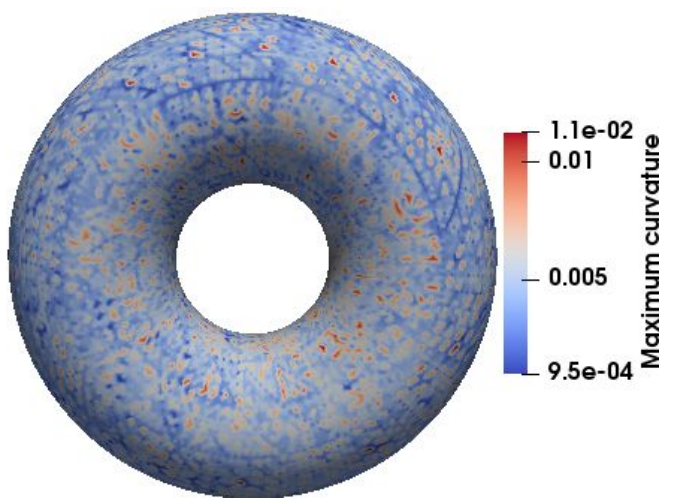

(d) NCC result

Fig. 3. Explicite Meshes Arising from Analytic Parametric Torus Surfaces.

The second triangular mesh reported in Fig. 2(b) is generated form the analytic sinusoidal parametric surface $(x, y, \sin (x) \cos (y))$ [9]. The analytic expression of the shape operator for the sinusoidal surface is represented by a not symmetric matrix. For the four comparative method, we first estimate the shape operator and then we compute the principal curvature represented by the two eigenvalues of each estimated operator.

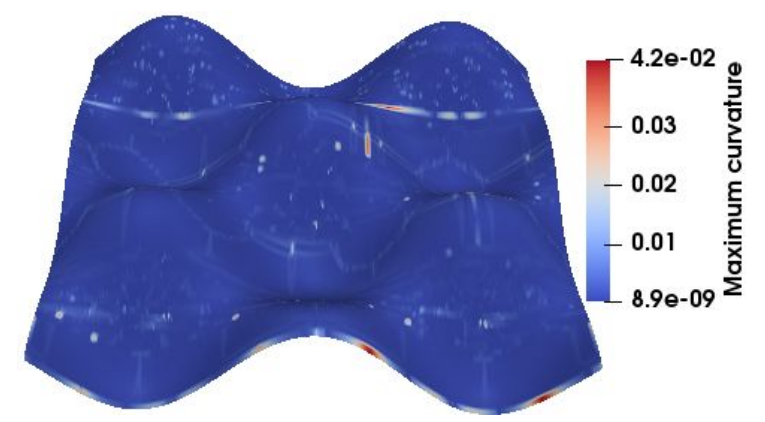

(a) $\mathrm{CBE}$ result

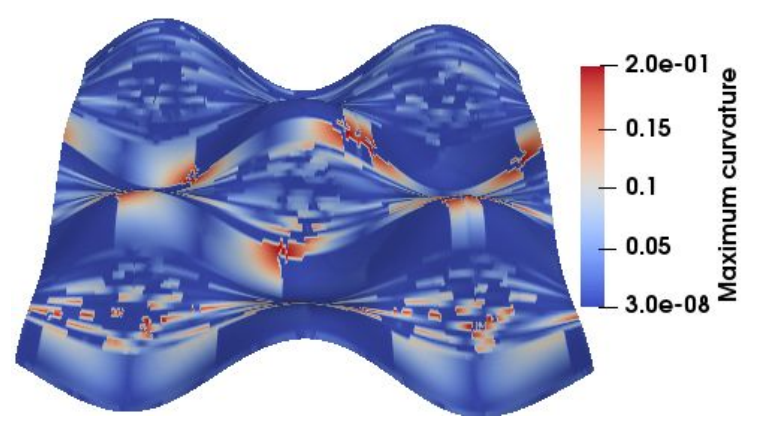

(b) DEM result

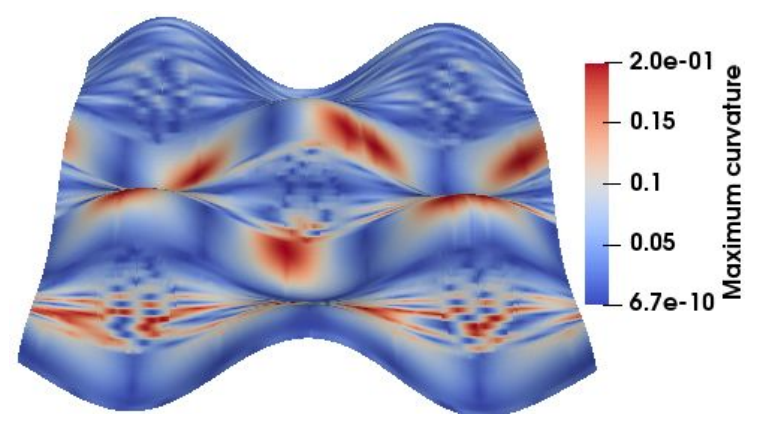

(c) MSC result

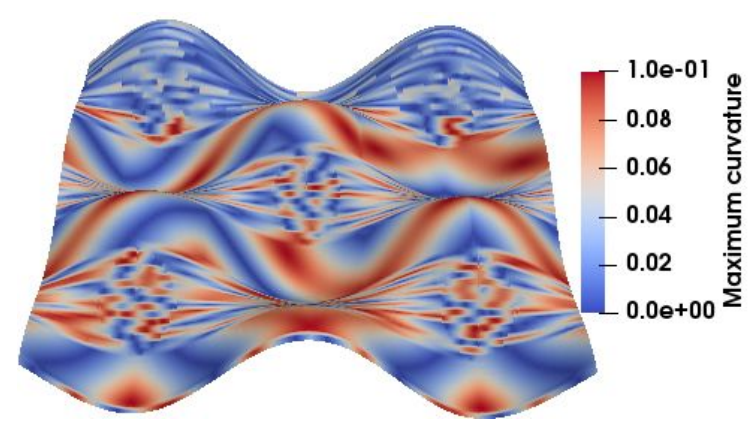

(d) NCC result

Fig. 4. Explicite Meshes Arising from Analytic Parametric Sinusoidal Surfaces.

The eigenvalue corresponding to the maximum curvature is then compared to the maximum curvature arising from 
the analytic expression of the shape matrix. For the torus mesh with symmetric shape operator, the absolute difference between the estimated and analytic maximum curvatures is reported in Fig. 3. As can clearly seen in Fig. 3(a), the lowest values of error difference between the exact and estimated maximum curvature is achieved by the proposed shape operator estimation method. For the sinusoidal mesh with unsymmetrical shape operator matrix, it can be clearly noticed that the proposed shape operator estimation method outperform the stat-of-art methods in term of accuracy. In Fig. 4(a), we notice the domination of blue color characterizing lowest error between estimated and exact curvature values. Also, due to the averaging shape operator estimation expression (8), the blue color is also uniformly distributed along the mesh. The

TABLE I. Absolute MEAN ERror Between Estimated and EXact MaXimum Curvature on Torus and Sinusoidal Meshes.

\begin{tabular}{lcr} 
& $\begin{array}{c}\text { Torus mesh } \\
\text { Symetric operator }\end{array}$ & $\begin{array}{c}\text { Sinusoidal mesh } \\
\text { Unsymetric operator }\end{array}$ \\
\hline CBE & $1.0210^{-4}$ & $2.0210^{-4}$ \\
DEM & $4.5010^{-4}$ & $6.7510^{-3}$ \\
MSC & $2.3210^{-2}$ & $3.4310^{-2}$ \\
CCM & $1.5010^{-3}$ & $5.5010^{-3}$ \\
\hline
\end{tabular}

absolute mean errors between estimated and exact maximum curvature on torus and sinusoidal meshes are reported in Table I. On the torus mesh with symmetric shape operator, a little difference between the proposed method and the DEM is noticed; however, for unsymmetrical shape operator arising from the sinusoidal surface, the proposed approach largely outperform all comparative methods. To evaluate convergence rate of the four shape operator estimation methods, we report in Fig. 5 the evolution of the mean error difference values in term of mesh resolution for the torus and sinusoidal meshes. In the case of torus mesh with symmetrical shape operator, we observe that the four methods have almost the same rat of convergence, with a small advantage of the proposed method. In contrast to the sinusoidal mesh with unsymmetrical shape operator, we can clearly distinguish the net performance the proposed CBM in comparison with the stat-of-art methods.

\section{B. Qualitative Evaluation}

The second set of evaluation tests concern two triangular meshes: the catenoid mesh generated by the javaiew software and the standard Fandisk meshes with sharp features reported in Fig. 6(b). The catenoid mesh reported in Fig. 6(a) is a revolution surface generated by rotating catenary curve about an axis [9], it is a minimal surface, which means that it occupies the least area when bounded by a closed space. A minimal surface is essentially characterized by a vanishing mean curvatures elsewhere, that is, $H(v)=0$ for each vertex $v$ of the mesh. Recall that the mean curvature is the average of the principles curvatures (7). In the Fig. 7, we report the absolute average mean curvature values achieved by the four estimation methods for the minimal catenoid surface mesh. We notice that the proposed Shape operator estimation methods presents the smallest absolute error value flowed together with the Multi-Scale Curvature (MSC) Estimation methods (CCM) and the Normal Cycle Curvature (NCC), the worst values ares achieved by the finite Difference curvature Estimation Method (DEM).
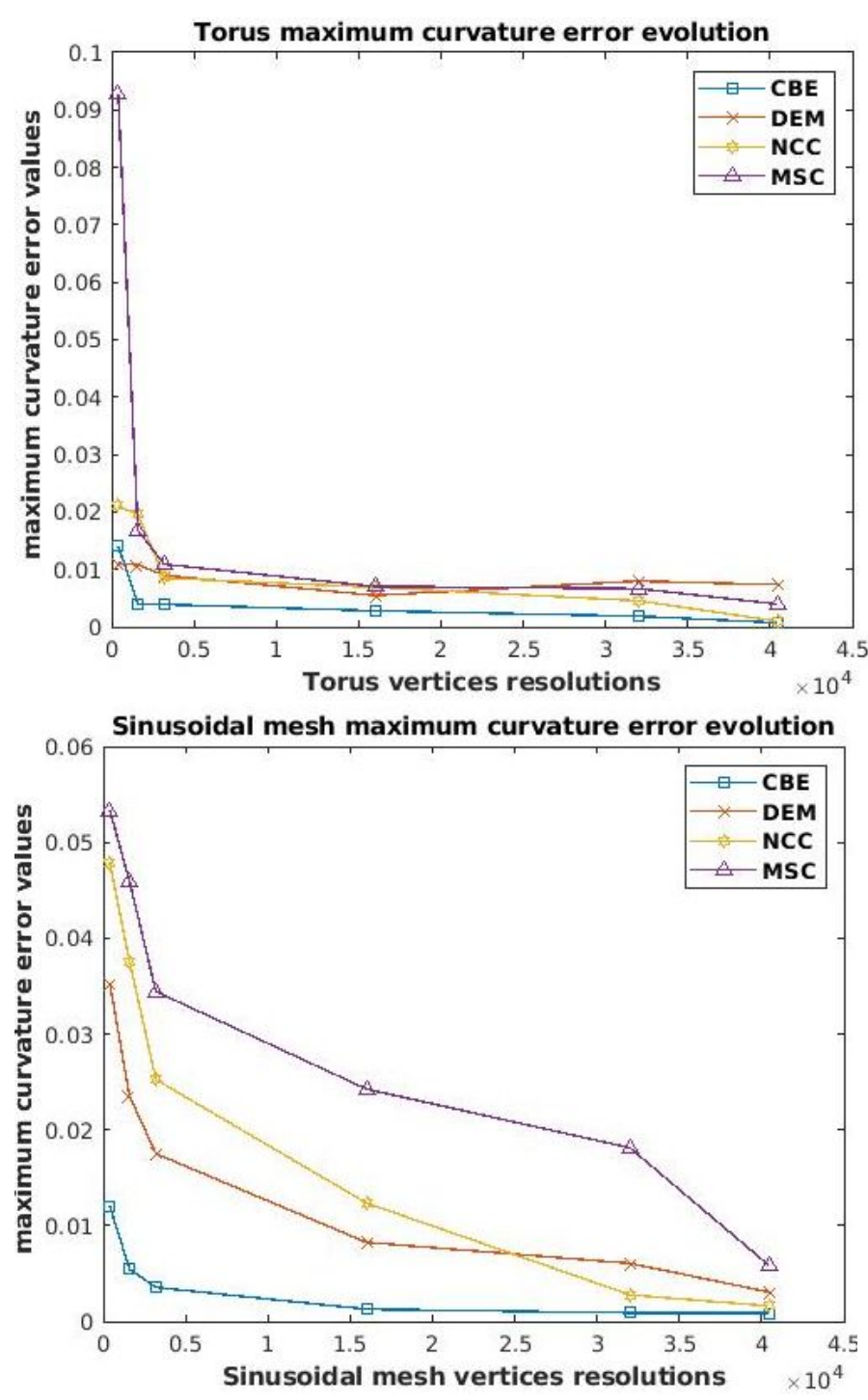

Fig. 5. Maximum Error Metric Evolution of Maximum Principal Curvatures Computed by the Comparative Estimation Methods on Torus and Sinusoidal Meshes.

In computer aided 3D design and mesh reprocessing applications, the maximum curvature is largely used to determine sharp features like edges and corners. Such geometric characteristics are characterized by a higher maximum curvature values. In Fig. 8 we consider a gain the catenoid and the fandisk meshes and we compute the maximum curvature values by the proposed shape operator estimation method. From the color map of maximum curvature values for the the catenoid and fandisk meshes depicted in Fig. 8(b), we observe that the sharp features like edges and corners are well identified by the proposed curvature estimation methods. The maximum curvature can also be used to detect defects that my arise during the fabrication process. The Fig. 8(a) shows the color map of the maximum curvature values for the catenoid mesh, we can clearly observe the crack along mesh that cannot be visually detected in the original object in Fig. 6(a). The experimental results show the effectiveness of the proposed mean value shape operator estimation method. 


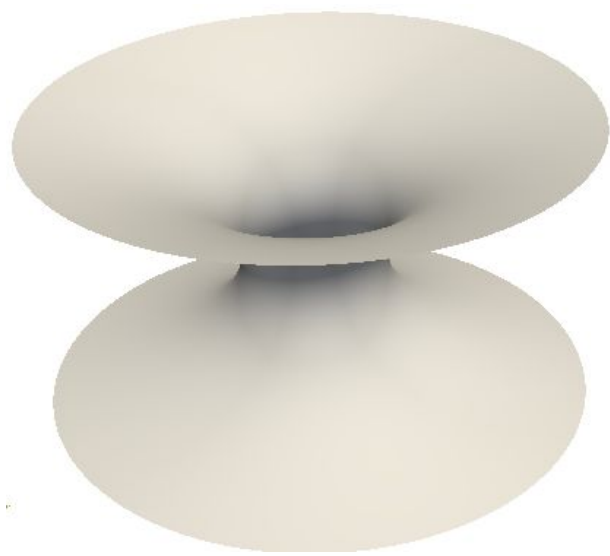

(a) Catenoid mesh

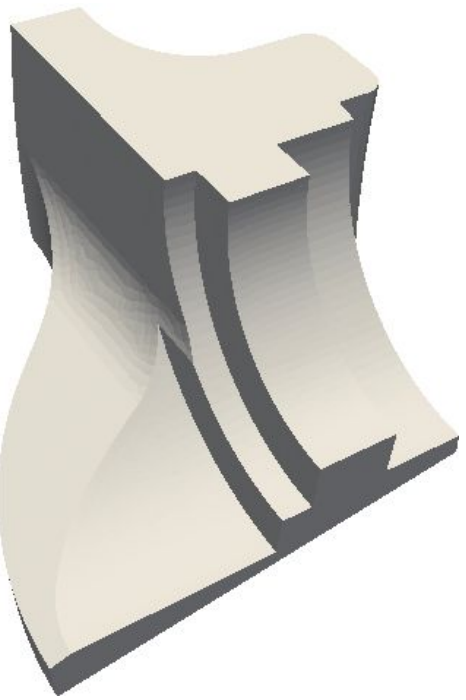

(b) Fandisk mesh

Fig. 6. Explicite Meshes Arising from Analytic Parametric Torus and Sinusoidal Surfaces.

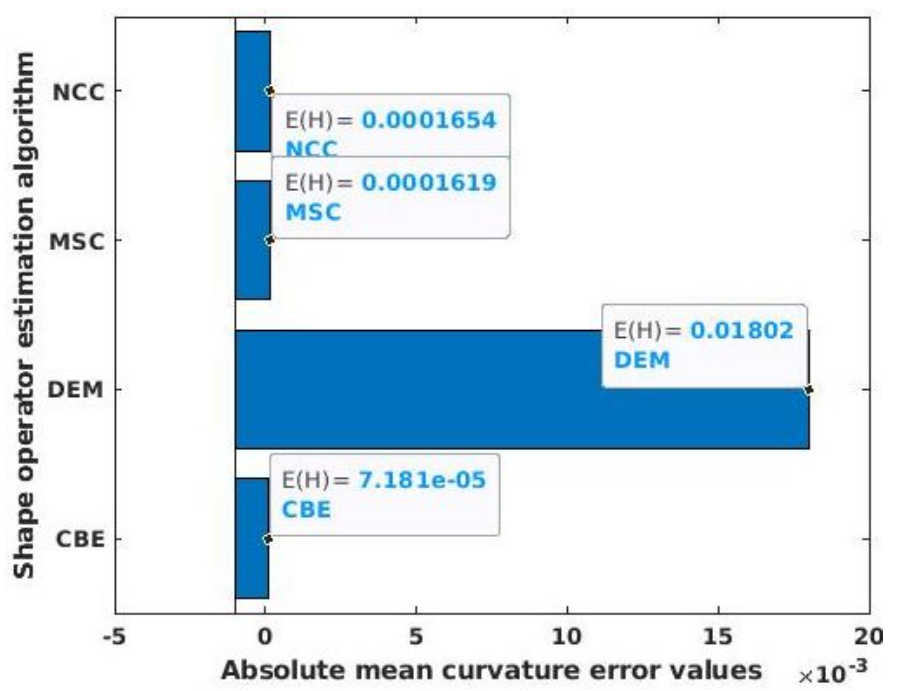

Fig. 7. Mean Curvature Errors Values for the Catenoid Minimal Surface Mesh.

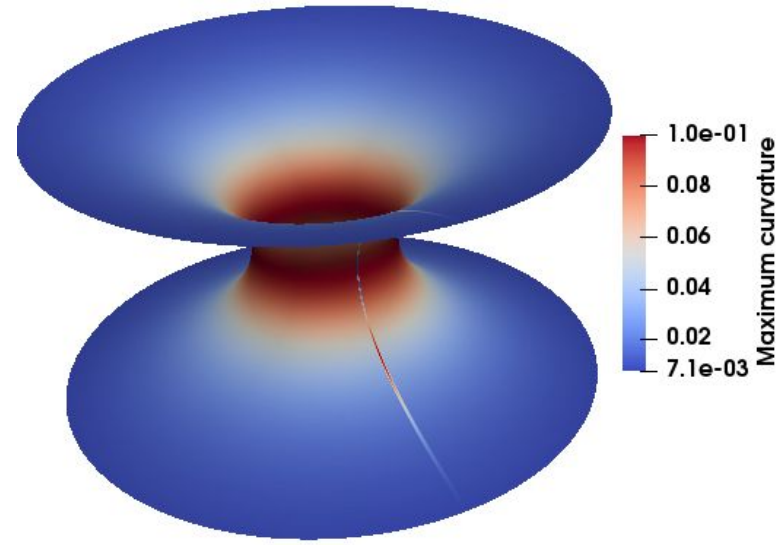

(a) Catenoid color map

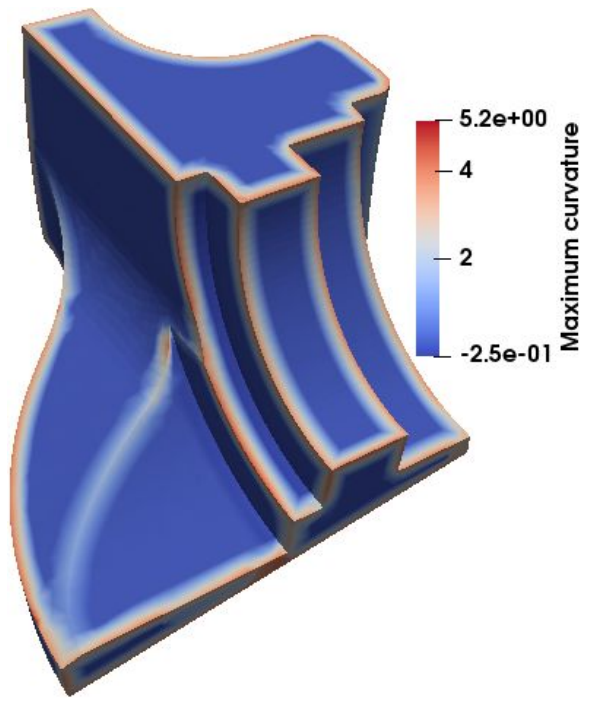

(b) Fandisk color map

Fig. 8. Maximum Curvature Color Map.

\section{Discussion}

The results show that the proposed operator performs better compared with outperformed some the stat-of-art shape operator estimation methods. It is clear from the above experiments that the shape operator estimation approaches based on the faces finite difference largely outperform the local surface fitting approaches. In future work we plan to adapt the proposed approach to estimate the shape operator method on noisy surface. Due to the complexity and irregularity of mesh data, the challenge is to build a mesh neural network to learn shape operator values directly from mesh data.

\section{CONCLUSION}

In this paper we proposed a mean value based approach to estimate the shape operator on the triangular meshes. In contrast to the state-of-art estimation methods that produce a symmetric shape operator matrix, the proposed algorithm proposed in this work is derived directly from the theoretical definition of the shape operator, and hence produced an estimation that mimetic the continues unsymmetrical nature of the shape operator. To demonstrate the performance of our approach, different tests on a variety of standard meshes are 
conducted by a quantitative and qualitative comparative study are presented.

\section{REFERENCES}

[1] Batagelo, H., Wu, ST. Estimating curvatures and their derivatives on meshes of arbitrary topology from sampling directions Visual Comput. 23, pp. 803-812 (2007). https://doi.org/10.1007/s00371-007-0133-8

[2] COHEN-STEINER, D., AND MORVAN, Restricted Delaunay Triangulations and Normal Cycle In Proc. Symposium on Computational Geometry (2003). https://doi.org/10.1145/777792.777839

[3] Demir, J. Kehrer and R. Westermann, Screen-space silhouettes for visualizing ensembles of 3D isosurfaces 2016 IEEE (PacificVis), (2016), pp. 204-208. doi: 10.1109/PACIFICVIS.2016.7465271.

[4] Deng H, Zhang W, Mortensen E, Dietterich T and Shapiro L Principal curvature-based region detector for object recognition, Computer IEEE. (2007),pp. 1-8. doi: 10.1109/CVPR.2007.382972

[5] do Carmo, M. P. Differential geometry of curves and surfaces, Prentice Hall,(1976).

[6] El Ouafdi A. F., El Houari H., Ziou D. Anisotropic adaptive method for tri.angular meshes smoothing IET Image Process. 9 (6), (2015), 516-526. doi: 10.1049/iet-ipr.2014.0447

[7] Evangelos Kalogerakis, Patricio Simari, Derek Nowrouzezahrai, and Karan Singh. Robust Statistical Estimation of Curvature on Discretized Surfaces. In Symposium on Geometry Processing. (2007), 13-22. doi: 10.2312/SGP/SGP07/013-022

[8] Grinspun, E., Gingold, Y., Reisman, J., Zorin, D Computing discrete shape operators on general meshes. Comput Graph. Forum, 25(3),(2006), 547-556. https://doi.org/10.1111/j.1467-8659.2006.00974.

[9] K. Khadem S., E.Preus and, Reitebuch U., Publication of Interactive Visualizations with JavaView, http://www.javaview.de/, retrieved in Mai 2021.

[10] Klaus Hildebrandt and Konrad Polthier. Generalized shape operators on polyhedral surfaces. Comput. Aided Geom. Des. 28(5), (2011), 321-343. doi: https://doi.org/10.1016/j.cagd.2011.05.001

[11] M. Liu, Y. Liu and K. Ramani, Anisotropic filtering on normal field and curvature tensor field using optimal estimation theory (SMI '07), (2007), 169-178. doi: 10.1109/SMI.2007.5.

[12] Mao Zhihong, Cao Guo, Ma Yanzhao , Kunwoo Lee Curvature estimation for meshes based on vertex normal triangles Comput. Aided Geom. Des., 43(12), (2011), 1561-1566 https://doi.org/10.1016/j.cad.2011.06.006

[13] Merigot Q, Ovsjanikov M, Guibas L. Robust Voronoi-based curvature and feature estimation, Geometric and physical modeling, (2009), 1-12. doi/10.1145/1629255.1629257
[14] Panozzo, D., Puppo, E., Rocca, L. Efficient multi-scale curvature and crease estimation 2nd International Workshop on Computer Graphics, Computer Vision and Mathematics, GraVisMa 2010 - Workshop Proceedings, (2010), 9-16.

[15] M. Prantl, L. Vása, I. Kolingerová Fast Screen Space Curvature Estimation on GPU Published in VISIGRAPP, (2016), $10.5220 / 0005676801490158$

[16] Tang, Y., Sun, X., Huang, D., Morvan, J.M., Wang, Y., Chen, L. 3D face recognition with asymptotic cones based principal curvatures IEEE (ICB), (2015) 466-472. doi: 10.1109/ICB.2015.7139111

[17] Tang, Y., Li, H., Sun, X. et al Principal Curvature Measures Estimation and Application to 3D Face Recognition J Math Imaging Vis 59, 211-233 (2017). https://doi.org/10.1007/s10851-017-0728-2

[18] TAUBIN, G. Estimating the Tensor of Curvature of a Surface from a Polyhedral Approximation, IEEE (ICCV), (1995), 902-907. doi: 10.1109/ICCV.1995.466840.

[19] H. Theisel, C. Rossi, R. Zayer and H. P. Seidel, Normal based estimation of the curvature tensor for triangular meshes 12th Pacific Conference on Computer Graphics and Applications, (2004), 288-297, doi: 10.1109/PCCGA.2004.1348359

[20] W.-S. Tong and C.-K. Tang, Robust estimation of adaptive tensors of curvature by tensor voting IEEE Trans. Pattern Anal. Mach. Intell., 27(3), (2005), pp. 434-449. doi:10.1109/TPAMI.2005.62

[21] Torsten Langer Alexander Belyaev Hans-Peter Seidel Exact and Interpolatory Quadratures for Curvature Tensor Estimation Comput. Aided Geom. Des. 24, (2007) 443-463, https://doi.org/10.1016/j.cagd.2006.09.006

[22] S. Rusinkiewicz, Estimating curvatures and their derivatives on triangle meshes Proceedings. 2nd International Symposium on 3D Data Processing, Visualization and Transmission, (2004), 486-493. doi: 10.1109/TDPVT.2004.1335277.

[23] S. Yoshizawa, A. Belyaev, H. Yokota and H. Seidel, Fast and Faithful Geometric Algorithm for Detecting Crest Lines on Meshes (PG'07), (2007), 231-237. doi: 10.1109/PG.2007.24.

[24] Szilvási-Nagy, M. Face-based Estimations of Curvatures on Triangle Meshes J. Geom. Graph. 12 (2008), 63-73.

[25] S. Sharma, S. Vinuchakravarthy and S. J. Subramanian Estimation of surface curvature from full-field shape data using principal component analysis 2016 IOP Publishing Ltd Measurement Science and Technology, Volume 28, Number 1 Citation Sameer Sharma et al 2017 Meas. Sci. Technol. 28015003

[26] Shifrin. T. DIFFERENTIAL GEOMETRY: A First Course in Curves and Surfaces, 2015,Lecture notes, University of Georgia, http://alpha.math.uga.edu/ shifrin/ShifrinDiffGeo.pdf. 